\title{
DIFICULDADES DAS LACTANTES FRENTE AO ALEITAMENTO MATERNO
}

\author{
DIFFICULTIES OF INFANTS IN FRONT OF BREASTFEEDING
}

${ }^{1}$ Ana Sylvia Castro Carvalho, ${ }^{2}$ Bruna Dulliny dos Santos, ${ }^{3}$ Juliana Lemos Schneid. \begin{abstract}
RESUMO
Introdução O aleitamento materno é reconhecido pela Organização Mundial da Saúde (OMS) como uma prática de amamentação ideal para a alimentação do bebê. Objetivo Geral: Identificar as possíveis dificuldades frente ao aleitamento materno com as lactantes. Material e Métodos: Trata-se de um estudo descritivo, realizado por meio de revisão integrativa de literatura. Utilizaramse artigos indexados nas bases de dados do Google Acadêmico, SciELO. Foram acessados 15 artigos, nos quais foram submetidos aos seguintes critérios de inclusão e exclusão respectivamente: artigos publicados entre os anos de 2000 à 2017, publicados em português, e excluídos artigos em inglês, fora do período descrito acima. Resultados e Discussão: É evidente o desmame precoce pelas nutrizes brasileiras, mas, com o intuito de promover a saúde materno-infantil nos últimos anos, aumentou-se o estimulo ao aleitamento materno por parte de profissionais. Conclusão: A amamentação deve ser o objetivo de todos os profissionais de saúde, que mantem um contato direto com puérperas e recém-nascidos. O enfermeiro tem um papel de fundamental importância dentro dos serviços de saúde, ao orientar sua equipe, quanto á assistência que deve ser prestada á esse público e as informações que serão repassadas, durante a consulta de enfermagem e pré-natal. Ficando evidente que as intercorrências mamaria como dor, fissuras, ingurgitamento mamário e mastite, são influenciadora direta para o desmame precoce.
\end{abstract}

Palavras-chave: Dificuldades, lactantes, aleitamento, materno.

\section{ABSTRACT}

Introduction: Breastfeeding is recognized by the World Health Organization (WHO) as an ideal breastfeeding practice for baby feeding. Objective: Identify the possible difficulties in breastfeeding with infants. Methodology: It is a descriptive study, carried out through an integrative literature review. Indexed articles were used in the databases of Google Aca-Démico, SciELO. Fifteen articles were accessed, in which they were submitted to the following inclusion and exclusion criteria respectively: articles published between the years 2009 to 2017, published in Portuguese, and excluded articles in English, outside the period described above. Results and discussion: Early weaning by Brazilian mothers is evident, but in order to promote maternal and child health in recent years, there has been an increase in breastfeeding stimulation by professionals. Final considerations: Breastfeeding should be the goal of all health professionals, who maintain direct contact with puerperae and newborns. Nurses play a fundamental role in the health services, when guiding their staff, regarding the assistance that should be provided to this public and the information that will be passed on during the nursing and prenatal consultation. It being evident that breast intercurrences such as pain, fissures, breast engorgement and mastitis are direct influencer for early weaning.

Key words: Difficulties, nursing, breastfeeding, maternal.

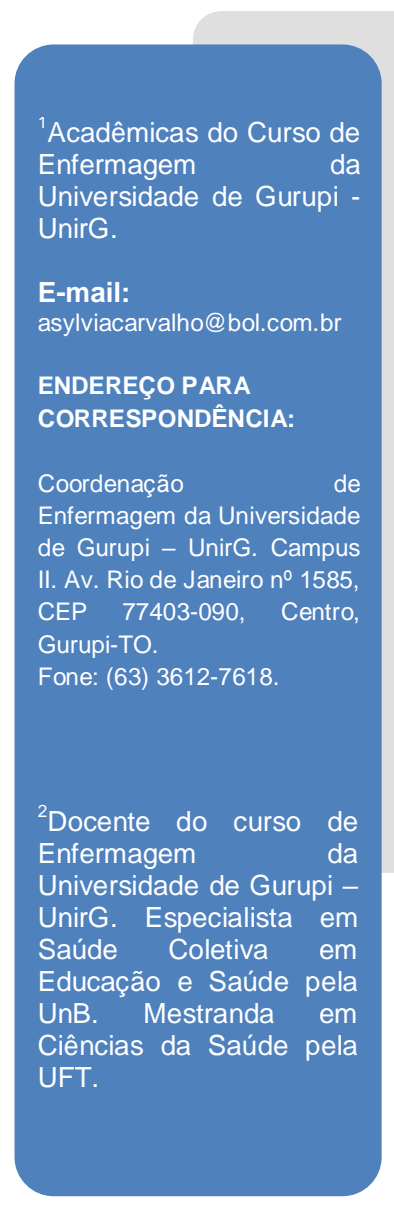

\section{INTRODUÇAOO}

O aleitamento materno exclusivo é reconhecido pela Organização Mundial da Saúde (OMS) como a melhor forma de amamentar a criança até o sexto mês de vida ${ }^{1}$.

Dentre os fatores de benefícios do leite materno para a criança destacam-se a sua maior digestibilidade, composição química balanceada, ausência de princípios alergênicos, proteção contra infecções além de baixo custo, desenvolvimento cognitivo e emocional, saúde física e psíquica da mãe $e^{2,3}$. Porém, amamentar vai além do ato de nutrir. Torna-se um processo que envolve uma interação profunda entre mãe e filho ${ }^{4}$.
Segundo a OMS e o Unicef, em torno de seis milhões de vidas de crianças estão sendo salvas a cada ano por causa do aumento das taxas de amamentação exclusiva. Assim, a mortalidade por doenças infecciosas é seis vezes maior em crianças menores de dois meses não amamentadas, diminuindo essa taxa à medida que a criança cresce ${ }^{5}$.

O leite materno contém anticorpos que ajudam a proteger contra doenças comuns da infância, a diarreia é uma dessas doenças, especialmente em crianças de classe baixa. É fundamental ressaltar que essa proteção pode baixar quando o aleitamento deixa de ser exclusivo. Dar ao bebê água ou chás, prática considerada 
Recebido: 05 junho 2018. Aceito: 14 dezembro 2018. Revista Amazônia Science \& Health - 2018 Out/Dez.
Carvalho, ASC. Santos, BD. Schneid, JL.

DIFICULDADES DAS LACTANTES FRENTE AO ALEITAMENTO MATERNO inofensiva para muitas mães, pode dobrar o risco de diarreia nos primeiros seis meses. A comercialização inadequada de substitutos do leite materno continua a comprometer os esforços para melhorar as taxas de amamentação e duração em todo o mundo ${ }^{6}$.

Em um estudo realizado pelo SISVAN, 2014, relata uma taxa de aleitamento materno exclusivo em crianças menores de 2 anos, no Tocantins com apenas $7.31 \%$ e no Brasil de $9.62 \%$. Relata ainda o aleitamento materno complementar no Tocantins de 23.74\%. A prevalência do aleitamento materno exclusivo em crianças menores de 6 meses, segundo as capitais e DF, regiões e Brasil, teve como resultados a região Norte com uma prevalência de $45,9 \%$, e Palmas com 35,7\%, Nordeste com 37,0\%, Cento-Oeste com 45,0\%, Sudeste com 39,4\%, Sul com 45,\% e Brasil com 41,0\% (OMS,2008)

Apesar de todas as comprovações científicas afirmando as vantagens do aleitamento materno exclusivo e dos esforços de diversos órgãos governamentais e de profissionais da saúde, as taxas de aleitamento materno no Brasil, ainda permanecem inferiores ao recomendado ${ }^{7}$. Assim, a baixa aderência ao aleitamento materno exclusivo constitui um sério problema de saúde pública. Diversos estudos apontam as fissuras, mamilos planos, dor, leite insuficiente, pega incorreta, ingurgitamento mamário e mastite como as principais dificuldades iniciais para o sucesso e manutenção do aleitamento materno exclusivo.

Diante do exposto o estudo teve como objetivo identificar as principais dificuldades encontradas pelas lactantes para realizar 0 aleitamento materno exclusivo até o sexto mês de vida da criança.

\section{METODOLOGIA}

Trata-se de um estudo descritivo, realizado por meio de revisão de literatura. Utilizaram-se artigos pesquisados através da ferramenta de busca do Google Acadêmico. Os artigos foram selecionados através da pesquisa dos seguintes descritores: "dificuldades amamentação", "aleitamento materno exclusivo", "benefícios do leite materno".

Foram selecionados 15 artigos. Como critérios de inclusão todas as categorias de artigo (original, revisão de literatura, reflexão, atualização, relato de experiência etc.); artigos com resumos e textos completos disponíveis para análise; aqueles publicados nos idiomas português, entre os anos 2000 e 2017, e artigos que contivessem em seus títulos e/ou resumos os seguintes descritores Os critérios de exclusão foram: estudos que não atendessem os critérios de inclusão mencionados e que não estivessem com texto completo.

\section{RESUTADOS E DISCUSSAO}

Independentemente dos diversos benefícios já conhecidos e claramente divulgados da amamentação e da formação de programas de encorajamento a essa prática, as taxas universais de aleitamento materno continuam inferior dos níveis indicados. Nesse contexto a tonificação das ações de apoio, promoção e proteção a amamentação exclusiva é essencial para o progresso dos índices da amamentação e redução das taxas de morbimortalidade infantil ${ }^{8}$.

As principais dificuldades encontradas pelas lactantes durante a amamentação foram à ocorrência de fissura, o ingurgitamento mamário, fissura mamilar, a dificuldade com a pega, a escassez de informações e orientações ${ }^{9}$. Estudos também sugerem que o fator cultural do "leite fraco" aliado às crenças maternas também interferem no sucesso da amamentação ${ }^{10}$.

\section{Trauma mamilar}

Efetuado em um hospital municipal de São Paulo, a porcentagem identificada para trauma mamilar $(39,2 \%)$ e fissura apareceram mais inferiores em demais estudos, contudo esses são os dois problemas mais mencionados na literatura como obstantes ou dificultadores do aleitamento materno ${ }^{11}$. Em outro estudo que apreciou a amamentação em HAC, em 2009, as dificuldades mais identificadas no primeiro monitoramento foram, também, o trauma mamilar $(84,3 \%)$ e a dor ao amamentar (72,3\%). ${ }^{12}$

A lesão mamilar é uma intercorrência mamária cuja incidência varia de 11 a 96\% nas lactantes durante a primeira semana depois do parto. Nota-se ainda que 80 a $95 \%$ destas mulheres queixam-se de incomodo nos mamilos e $26 \%$ apresentam dor extrema, contribuindo negativamente para a duração da amamentação. A principal consequência dessa dificuldade é a interrupção precoce da amamentação e o estímulo ao uso de mamadeira, o que leva ao desmame por uso de bicos artificiais. Nesse sentido, o trauma mamilar tem sido indicado como uma ocorrência decorrente do posicionamento e pega incorretos da criança durante o aleitamento materno, causando dor ao amamentar. Pois, quando o bebe tem urna boa pega, o mamilo fica em uma posição dentro da boca da criança que o protege da fricção e compressão, prevenindo assim, lesões mamilares. Por esse ângulo, a conduta mais importante para sua diminuição é a educação das mulheres, desde a gestação, em relação à técnica correta de amamentação $^{13}$.

\section{Pega incorreta}


Recebido: 05 junho 2018. Aceito: 14 dezembro 2018. Revista Amazônia Science \& Health - 2018 Out/Dez.
Carvalho, ASC. Santos, BD. Schneid, JL.

DIFICULDADES DAS LACTANTES FRENTE AO ALEITAMENTO MATERNO
De acordo com Amaral et al $^{14}$ dentre as dificuldades apontadas pelas mulheres no ato de amamentar, a relutância de pega da mama por parte da criança e a preferência por uma mama em detrimento a outra foi relatada. A resistência dos bebês ao serem amamentados ao seio pode estar ligada ao uso de bicos artificiais ou mamadeira, ou ainda ao surgimento de dor ao ser posicionado para mamar. Assim a suspensão destes artefatos, quando presentes, o posicionamento adequado, a insistência nas mamadas, além da tranquilidade materna consistem em manejos importantes para estimular o bebê.

$\mathrm{Na}$ pega correta, o bebê faz a abertura extensa da boca, pegando não apenas o mamilo, mas também parcela da aréola, e gerando um lacre ideal entre as estruturas orais e a mama. Para a criação desse lacre, na parte anterior os lábios estão invertidos externamente, (sendo que o lábio superior e a língua são os prudentes responsáveis por um vedamento apropriado), e a língua se amparam na gengiva inferior, curvando-se para cima em contato com a mama. A função do lacre equivale no desenvolvimento do vácuo intra-oral, criado por movimentos da mandíbula agregado a movimentos das das bochechas, coxins de gorduras. A posição inadequada da genitora e/ou do bebê no aleitamento materno complica a correta posição da boca do bebê em relação ao complexo aréolo-mamilar, sucedendo no que se designa de má pega. A má pega, por sua vez, envolve-se na prática de extração e sucção do leite, sendo capaz de criar dor e desconforto para a genitora e traumas mamilares, complicando até mesmo a sucessão do aleitamento, caso não seja adequadamente corrigida $^{15}$. Diante disso, entende-se que 0 acompanhamento e orientação da amamentação, principalmente por parte do enfermeiro, pois este segundo Demitto et $\mathrm{al}^{16} \mathrm{em}$ pesquisa de revisão de literatura sobre o atendimento pré-natal cita que vários estudos destacaram para a multidimensionalidade do desempenho desse profissional, enfatizando-se, além da atuação e conhecimento técnico, a interação entre ele e as gestantes e também com os demais membros da equipe, e como é estabelecido um vínculo de confiança por parte das gestantes 0 que proporciona o aumento da frequência nas consultas de pré-natal e melhora na assistência de saúde prestada a esse público. Ressalta-se também que programas de promoção e incentivo a amamentação fazem parte da capacitação da equipe de saúde, proporcionando grande impacto nas práticas desses profissionais, ocasionando maior duração da amamentação nas comunidades por eles assistidas ${ }^{17}$. Assim, prioriza-se a importância do profissional de enfermagem, pois ele desenvolve uma melhor assistência voltada às gestantes e puérperas, não apenas para diminuir os altos índices de desmame, mas, sobretudo, com a função de orientar e tornar este ato uma experiência saudável e prazerosa para ambos ${ }^{18}$.

\section{Ingurgitamento mamário}

Entre as várias dificuldades enfrentadas pelas nutrizes, 0 ingurgitamento mamário destaca-se como um dos problemas mais frequentes nas unidades de puerpério, que pode influenciar de maneira negativa na continuidade da amamentação ${ }^{19}$.

É necessário diferenciar o ingurgitamento fisiológico, que é normal, do patológico. O primeiro apesar de discreto, significa que o leite está "descendo", não sendo necessária qualquer intervenção. Já no ingurgitamento patológico, a mama fica excessivamente distendida, causando grande desconforto, podendo está acompanhado de febre e mal-estar. Pode haver áreas difusas avermelhadas, edemaciadas e brilhantes, dificultando a pega do bebê, dificultando a saída do leite. Leite em abundância, início tardio da amamentação, mamadas infrequentes, restrição da duração e frequência das mamadas e sucção ineficaz do bebê favorecem o aparecimento do ingurgitamento. Portanto, em relação ao manejo e prevenção para 0 ingurgitamento mamário, recomenda-se amamentação em livre demanda, iniciada o mais cedo possível, preferencialmente logo após o parto, e com técnica correta, e o não uso de complementos (água, chás e outros leites) sendo estas, medidas eficazes ${ }^{20}$.

\section{Mastite}

A mastite da lactação ou puerperal é um processo infeccioso agudo das glândulas da mama que ataca mulheres em estágio de lactação, acontece mais geralmente na segunda e terceira semanas após o parto e dificilmente após a $12^{\mathrm{a}}$ semana, com achados clínicos que vão a partir da inflamação focal, com sintomas sistêmicos como prostração, astenia, septicemia calafrios, febre, abscessos e mal-estar geral. Devido a dor e ao desconforto, bem como confiarem que o leite da materno afetada vai gerar um mal ao bebê, muitas genitora desmamam precocemente os seus bebês, se não conduzirem corretamente na orientação e forem apoiadas. A mastite, quando não tratada antecipadamente, pode progredir para abscesso. $O$ mais adequado tratamento é o acrescimento de líquidos, massagem, seguida de ordenha, aplicação de calor e/ou frio e repouso. A massagem ajuda a fluidificação do leite por passagem de energia cinética, empregue para quebra das interações intermoleculares que se estabelecem no leite acumulado na parte interna da mama, além de incitar a síntese de ocitocina essencial ao reflexo de ejeção do leite. Pode ser fundamental a utilização de antibióticos, antitérmicos e analgésicos ${ }^{21}$. 
Recebido: 05 junho 2018. Aceito: 14 dezembro 2018. Revista Amazônia Science \& Health - 2018 Out/Dez.
Carvalho, ASC. Santos, BD. Schneid, JL.

DIFICULDADES DAS LACTANTES FRENTE AO ALEITAMENTO MATERNO

\section{Cultura e tabus - o "leite fraco"}

O mito do leite fraco, nos dias de hoje, é um dos principais motivos que leva a complementação precoce. A aparência aguada do leite materno do colostro faz com que a lactante julgue seu leite como fraco, não suprindo assim, as necessidades do bebê ${ }^{22}$. Este conhecimento errado consegue está ligado a ausência de informações das mães quanto aos benefícios do seu leite, em relação como o leite materno é gerado e ao ato de associarem o choro dá criança à falta de alimentação, o que nem sempre é verídico. A cultura prejudica vigorosamente nas crenças maternas e a interferência de outras pessoas (avós, vizinhas) no que tange ao leite fraco, é capaz de levar as mães a confiarem que não são aptas de gerar leite em quantia suficiente, mesmo quando são orientadas ${ }^{23}$. A aparência aguada do leite materno faz com que a mãe considere seu leite inferior, acreditando que não serve para atender as necessidades do bebê por apresentar diferenças do leite popularmente conhecido como leite forte - o leite de vaca ${ }^{24}$. Destaca-se que a prática alimentar da nutriz está permeada de tabus, crenças e mitos, sendo que os alimentos permitidos e proibidos têm como objetivo principal aumentar a produção e a qualidade do leite materno. Portanto, para as mulheres-mães uma alimentação inadequada, fraca pode ocasionar a redução da quantidade e da qualidade do leite.

\section{Falta de informação}

Outros fatores colaboram para o desmame precoce, no entanto, a ausência de conhecimento sobre aleitamento materno por parte das mães tem desempenhado papel significativo na redução da duração da amamentação. Contudo, as distorções de informações sobre o aleitamento materno, os significados que a mulher atribui ao aleitamento materno e as crenças representam maior influência na duração da amamentação. Assim, acredita-se que investigar o conhecimento das mães sobre o aleitamento materno pode favorecer no direcionamento na reorientação das práticas adotas pelos profissionais de saúde e ações educativas. A educação e a preparação das mulheres para a lactação durante 0 período pré-natal comprovadamente colabora para o sucesso do aleitamento materno, em especial entre as primíparas ${ }^{25}$.

Prioriza-se a importância do profissional de enfermagem, pois ele amplifica uma melhor assistência voltada às gestantes e puérperas, não apenas para diminuir os altos índices de desmame, mas, sobretudo, com a função de orientar e tornar este ato uma experiência saudável e prazerosa para ambos $^{26}$.

\section{CONCLUSAOO}

Mesmo com toda excelência do aleitamento materno exclusivo e das orientações através de ações de educação em saúde na comunidade e consulta do pré-natal pelos profissionais da saúde a prevalência ainda é baixa de aleitamento materno exclusivo em nosso país. Sabe-se que o leite materno contém todos os nutrientes essenciais para o desenvolvimento e crescimento da criança, sendo capaz de suprir sozinho as necessidades nutricionais da criança até o sexto mês de vida, e diminui a chance de ter diversas doenças. Porém, ainda é necessário novas estratégias para o encorajamento do aleitamento materno exclusivo para que tenha um aumento nas taxas de aleitamento, bem como redução da morbimortalidade das crianças.

O papel do profissional da saúde, bem como detectar e ajudar a prevenir possíveis intercorrência como a pega e a posição de amamentar, que geram prováveis complicações mamilares como dor, fissuras, ingurgitamento e mastite, fica evidente que as intercorrências mamaria são influenciadora direta para o desmame precoce.

O enfermeiro tem um papel de fundamental importância dentro dos serviços de saúde, ao orientar sua equipe, quanto á assistência que deve ser prestada á esse público e as informações que serão repassadas, durante a consulta de enfermagem e pré-natal. Por tanto é necessário que a equipe de saúde promova ações de proteção e apoio a amamentação para obter o sucesso na pratica do aleitamento materno exclusivo.

\section{REFERENCIAS}

1 BRASIL. Ministério da Saúde. Secretaria de Atenção à Saúde. Departamento de Atenção Básica. Saúde da Criança: nutrição infantil: aleitamento materno e alimentação complementar. Brasília: Editora do Ministério da Saúde; 2009.

2 BUENO, KCV. A importância do aleitamento materno exclusivo até os seis meses de idade para a promoção de saúde da mãe e do bebê. Minas Gerais; 2013.

3 BRASIL. Ministério da Saúde. Secretaria de Atenção à Saúde. Departamento de Atenção Básica. Saúde da Criança: nutrição infantil: aleitamento materno e alimentação complementar. Brasília: Editora do Ministério da Saúde; 2009.

4 BRASIL. Ministério da Saúde. Secretaria de Atenção à Saúde. Departamento de Atenção Básica. Saúde da Criança: nutrição infantil: aleitamento materno e alimentação complementar. Brasília: Editora do Ministério da Saúde; 2009. 
Recebido: 05 junho 2018. Aceito: 14 dezembro 2018. Revista Amazônia Science \& Health - 2018 Out/Dez.
Carvalho, ASC. Santos, BD. Schneid, JL.

DIFICULDADES DAS LACTANTES FRENTE AO ALEITAMENTO MATERNO
5 BRASIL. Ministério da Saúde. Secretaria de Atenção à Saúde. Departamento de Atenção Básica. Saúde da Criança: nutrição infantil: aleitamento materno e alimentação complementar. Brasília: Editora do Ministério da Saúde; 2009.

6 PASSANHA A et al. Elementos protetores do leite materno na prevenção de doenças gastrintestinais e respiratórias. Rev. Bras. Cresc. e Desenv.; 2010.

7 FRANCO SC et al. Aleitamento materno exclusivo em lactentes atendidos na rede pública do município de Joinville. Recife: Rev. Bras. Saúde Mater. Infantil; 2008.

8 ROCCI E, FERNANDES RAQ. Dificuldades no aleitamento materno e influência no desmame precoce. Revista Brasileira de Enfermagem [Internet]. 2014; 67(1):22-27. Recuperado de: http://www.redalyc.org/articulo.oa?id=267030130003

9 MORENO BB, SCHIMIDT KT. Aleitamento Materno e Fatores Relacionados ao Desmame Precoce. Paraná: Cogitare Enfermagem; 2014.

10 ROCCI E, FERNANDES RAQ. Dificuldades no aleitamento materno e influência no desmame precoce. Revista Brasileira de Enfermagem [Internet]. 2014; 67(1):22-27. Recuperado de: http://www.redalyc.org/articulo.oa?id=267030130003

11 ROCCI E, FERNANDES RAQ. Dificuldades no aleitamento materno e influência no desmame precoce. Revista Brasileira de Enfermagem [Internet]. 2014; 67(1):22-27. Recuperado de: http://www.redalyc.org/articulo.oa?id=267030130003

12 FIGUEIREDO SF. Avaliação da iniciativa Hospital Amigo da Criança na prática do Aleitamento Materno exclusivo nos primeiros seis meses de vida em uma maternidade pública da cidade de São Paulo. Dissertação [Mestrado em Enfermagem]. São Paulo: Universidade Federal de São Paulo; 2009.

13 COCA KP, GAMBA MA et al. Fatores associados ao trauma mamilar na maternidade. Porto Alegre: Jornal de Pediatria; 2009.

14 Amaral LJX, Sales SS, Carvalho DPSRP, Cruz GKP, Azevedo IC, Ferreira Júnior MA. Fatores que influenciam na interrupção do aleitamento materno exclusivo em nutrizes. Revista Gaúcha de Enfermagem; 2015.

15 SANCHES MTC. Manejo clínico das disfunções orais na amamentação. Jornal de Pediatria. 2004; 80(5).
16 DEMITTO $\mathrm{MO}$ et al. Orientações sobre amamentação na assistência pré-natal: ua revisão integrativa. Sistema de Información Cientifíca; 2010.

17 LANA APB et al. Impacto de um programa para promoção da amamentação em um centro de saúde. Rio de Janeiro: Jornal de Pediatria; 2004

18 SOBRINHO et al. Dificuldades encontradas no aleitamento materno, sob a visão da Enfermagem. Sergipe: Universidade Tiradentes; 2017.

19 CASTRO KF, GARCIA TR et al. Intercorrências mamárias relacionadas à lactação: estudo envolvendo puérperas de uma maternidade pública de João Pessoa, PB. São Paulo: O Mundo da Saúde; 2009.

20 GIUGLIANI ERJ. Problemas comuns na lactação e seu manejo. Rio de Janeiro: Jornal de Pediatria; 2004.

21 SALES AN, VIEIRA, GO et al. Mastite Puerperal: Estudo de Fatores Predisponentes. Bahia: RBGO; 2000.

22 MARQUES ES et al. Mitos e crenças sobre o aleitamento materno. Minas Gerais: Departamento de Nutrição e Saúde; 2011.

23 ROCCI E, FERNANDES RAQ. Dificuldades no aleitamento materno e influência no desmame precoce. Revista Brasileira de Enfermagem [Internet]. 2014; 67(1):22-27. Recuperado de: http://www.redalyc.org/articulo.oa?id=267030130003

24 MARQUES ES, COTTA RMM, ARAÚJO RMA. Representações sociais de mulheres que amamentam sobre a amamentação e o uso de chupeta. Revista Brasileira de Enfermagem. 2009; 62(4):562-569. Recuperado de: http://www.redalyc.org/articulo.oa? $\mathrm{id}=267019598012$

25 SILVA NM, WATERKEMPER $R$ et al. Conhecimento de puérperas sobre amamentação exclusiva. Rio Grande do Sul: Revista Brasileira de Enfermagem; 2014.

26 SOBRINHO et al. Dificuldades encontradas no aleitamento materno, sob a visão da Enfermagem. Sergipe: Universidade Tiradentes; 2017. 